Recepción: 05 / 01 / 2018

Aceptación: 20 / 02 / 2018

Publicación: 24 / 04 / 2018

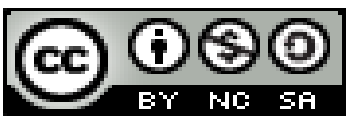

Ciencias de la Salud

\title{
Tratamiento neurorehabilitador integral en pacientes con esclerosis múltiple
}

\author{
Comprehensive neurorehabilitation treatment in patients with multiple sclerosis
}

\section{Tratamento abrangente de neurorreabilitação em pacientes com esclerose múltipla}

\author{
Mayra M. León-Cadme ${ }^{\mathrm{I}}$ \\ mayramercedes@hotmail.com \\ Mercy T. Sancan-Moreira II \\ mercy_sancan@hotmail.com \\ Diego J. Cuichán-Nuñez III \\ jota_tati@hotmail.com
}

\section{Correspondencia: mayramercedes@hotmail.com}

${ }^{I}$ Magister en Neuro Rehabilitación, Licenciada en Terapia Ocupacional, Tecnóloga Médica en Terapia Ocupacional, Docente de la Universidad Laica Eloy Alfaro de Manabí, Manta, Ecuador.

${ }^{\text {II }}$ Magister en Gerencia Educativa, Diploma Superior en Educación Universitaria por Competencias, Licenciada en la Especialización de Terapia Ocupacional, Tecnólogo Medico Especialización Terapia Ocupacional, Docente de la Universidad Laica Eloy Alfaro de Manabí, Manta, Ecuador.

III Magister en Seguridad y Salud Ocupacional, Licenciado en Terapia Ocupacional, Docente de la Universidad Laica Eloy Alfaro de Manabí, Manta, Ecuador. 


\section{Resumen}

El reconocimiento del entorno social y familiar con el que interactúan los pacientes diagnosticados con Esclerosis Múltiple suele incidir de forma positiva en las mejorías de la enfermedad. Ante esta posición, en este artículo se propone un estudio del tratamiento neurorehabilitador integral en pacientes con esclerosis múltiple, para ello se utilizó una metodología de tipo observacional y descriptivo de campo. La población estuvo constituida por 30 pacientes con diagnóstico de Esclerosis Múltiple previamente confirmada por el neurólogo, atendidos en el Servicio de Medicina Física y Rehabilitación del Hospital de Especialidades Eugenio Espejo de la ciudad de Quito Ecuador. Para su tratamiento se solicitó el consentimiento informado a los pacientes y familias y los criterios de inclusión, eran pacientes con edades de adultez mayores a 20 años. Los datos se registraron en una lista de coteo, entre los resultados de su análisis se constató mejorías en los pacientes con este padecimiento después de un programa de neuro-rehabilitación integral, independiente de la modalidad definida de presentación para el inicio de sus terapias y la evolución de la EM, manifiestos en una mejora de su condición física y con factores relacionados a su calidad de vida.

Palabras clave: esclerosis múltiple; neurorehabilitación integral; calidad de vida.

\section{Abstract}

The recognition of the social and family environment with which patients diagnosed with Multiple Sclerosis interact tends to have a positive effect on the improvement of the disease. Given this position, this article proposes a study of the integral neurorehabilitation treatment in patients with multiple sclerosis, for which an observational and descriptive field methodology was used. The population consisted of 30 patients diagnosed with Multiple Sclerosis previously confirmed by the neurologist, attended at the Physical Medicine and Rehabilitation Service of the Specialties Hospital Eugenio Espejo in the city of Quito, Ecuador. For their treatment, informed consent was requested to patients and families and the inclusion criteria were patients with adult ages over 20 years. The data were recorded in a checklist, among the results of their analysis, improvements were found in patients with this condition after a comprehensive neurorehabilitation program, independent of the defined modality of presentation for the initiation of 
their therapies and the evolution of MS, manifested in an improvement of their physical condition and factors related to their quality of life.

Keywords: multiple sclerosis; Comprehensive neurorehabilitation; quality of life.

\section{Resumo}

O reconhecimento do ambiente social e familiar com o qual os pacientes diagnosticados com Esclerose Múltipla interagem tende a ter um efeito positivo na melhora da doença. Diante dessa posição, este artigo propõe um estudo do tratamento da reabilitação neurorresistente integral em pacientes com esclerose múltipla, para o qual foi utilizada uma metodologia de campo observacional e descritiva. A população foi constituída por 30 pacientes diagnosticados com esclerose múltipla anteriormente confirmada por neurologista, serviu no Departamento de Medicina Física e Reabilitação Specialist Hospital Eugenio Espejo de Quito Equador. Para o seu tratamento, foi solicitado consentimento informado aos pacientes e familiares e os critérios de inclusão foram pacientes com idade adulta acima de 20 anos. Os dados foram registrados em uma lista de coteo, entre os resultados de suas melhorias de análise em pacientes com esta condição que foi encontrado depois de um programa abrangente de neuro-reabilitação independente do modo de exibição definida para o início de suas terapias e evolução da SM, manifestando-se em melhora de sua condição física e fatores relacionados à sua qualidade de vida.

Palavras chave: esclerose múltipla; Neurorreabilitação abrangente; qualidade de vida.

\section{Introducción}

El objetivo principal de la rehabilitación es mejorar la calidad de vida, disminuyendo las limitaciones en las actividades de la vida diaria, así como la restricción en la participación social, el mismo debe hacerse mediante un tratamiento integral e interdisciplinar basado en los problemas que se encuentra el paciente día a día derivados de la enfermedad, se trata de conseguir que la recuperación neurológica vaya siempre acompañada de la recuperación funcional. En el caso de la Esclerosis Múltiple (E.M), que es una enfermedad inflamatoria, desmielinizante e inmunológica que afecta al Sistema Nervioso Central y constituye la causa más frecuente de invalidez neurológica no traumática en el adulto joven. (Terré y Orient, 2007). 
La EM es una enfermedad muy variable y los síntomas dependen de las áreas del sistema nervioso central que se hayan visto afectadas y la magnitud del brote. Los episodios pueden durar días, semanas o meses e ir seguidos de períodos de reducción o ausencia de los síntomas (remisiones). También pueden presentarse de forma progresiva la fiebre, los baños calientes, la exposición al sol y el estrés pueden desencadenar o empeorar los brotes. No hay una EM típica. La mayoría de las personas con EM experimentan un conjunto diferente de síntomas, y aunque hay síntomas comunes a muchas personas, éstos pueden variar en severidad y duración, incluso en la misma persona. La fatiga es el síntoma más común y molesto a medida que la EM progresa. Mientras que algunos de estos síntomas son evidentes, otros tales como la fatiga, los problemas sensitivos, y los de memoria y concentración son a menudo síntomas ocultos. Estos pueden ser difíciles de describir a los demás y a veces los familiares y cuidadores no entienden los efectos que éstos tienen sobre la persona con EM, su empleo, actividades sociales y calidad de vida. Con base a lo expuesto, se presenta la caracterización del tratamiento neurorehabilitador integral en pacientes con esclerosis múltiple.

\section{Tipos de Esclerosis Múltiple}

Algunas personas se ven mínimamente afectadas por la enfermedad, y en otras avanza rápidamente hacia la incapacidad total; pero la mayoría de afectados se sitúa entre los dos extremos. Si bien cada persona experimentará una combinación diferente de síntomas de EM, hay varias modalidades definidas de presentación y evolución de la EM, entre las que se pueden mencionar, según la EME (2013):

Esclerosis Múltiple recurrente-remitente (EMRR): En esta forma de EM se producen períodos de recaída (brotes) seguidos de etapas de estabilización de duración variable (días o meses). Estas recaídas consisten generalmente en la aparición de síntomas neurológicos debidos a una nueva lesión en el sistema nervioso central. Tras el brote puede haber una remisión parcial de los síntomas e incluso una recuperación total. La enfermedad puede permanecer inactiva durante meses o años. Es el tipo más frecuente afectando inicialmente alrededor del $85 \%$ de las personas con EM.

Esclerosis Múltiple progresiva secundaria (EMPS): En algunas personas que tienen inicialmente EM con recaídas y remisiones, se desarrolla posteriormente una incapacidad 
progresiva en el curso de la enfermedad, frecuentemente con recaídas superpuestas y sin períodos definidos de remisión. Entre un 30 y un $50 \%$ de los pacientes que sufren inicialmente la forma recurrente-remitente de la EM, desarrollan la forma secundaria progresiva.

Esclerosis Múltiple progresiva primaria (EMPP): Esta forma de EM se caracteriza por la ausencia de brotes definidos, con un comienzo lento y un empeoramiento constante de los síntomas y de la discapacidad. Aproximadamente el 10\% de las personas con EM son diagnosticados con esta forma progresiva primaria.

Esclerosis Múltiple progresiva recidivante (EMPR): Es la forma menos común, dándose en un $5 \%$ de los casos. Se caracteriza por una progresión constante y sin remisiones desde el comienzo con una clara superposición de brotes con o sin recuperación completa.

\section{Equipos y recursos para el tratamiento integral de la esclerosis múltiple}

El manejo actual de la esclerosis múltiple, no se concibe sin el tratamiento por un equipo interdisciplinario efectivo desde el aspecto clínico, rehabilitatorio, psicológico y social.

El equipo debe incluir, plantea Plaja (2002):

- Médico Neurólogo y/o Médico Internista para el Tratamiento clínico farmacológico integral.

- Médico Rehabilitador o Fisiatra, el cual realizará la evaluación de cada caso en particular, indicará el tratamiento dependiendo del estadio y condiciones clínicas de cada paciente (espasticidad, parálisis, paresia, flacidez, etc.) y realizará el control y seguimiento con revaloración del paciente por lo menos cada bloque de 10 sesiones indicadas, realizando escalas de medición funcional como indicadores de evolución y mejoría respecto al inicio del tratamiento fisiátrico.

- Terapistas físicos, que realizarán el programa de ejercicios indicado por el médico rehabilitador.

- Terapistas ocupacionales, los cuales ayudarán a la realización de entrenamiento de las actividades de la vida diaria. 
- Terapista de lenguaje en caso de afectación a articulación del lenguaje.

- Psicólogos para psicoterapia de apoyo, manejo de crisis depresiva, de ansiedad, etc.

- Enfermeras con entrenamiento y orientación para el manejo de pacientes con esclerosis múltiple, enfermeras en rehabilitación.

- Trabajadoras sociales. Evaluación de entorno familiar.

- Técnicos ortesistas para la confección de férulas y/o aditamentos en casos requeridos.

Los recursos deberán incluir, plantean Ruiz, Martín, Delgado, García y Penche (2006), unidades de rehabilitación que cuenten con Servicio de Hospitalización, sobre todo para pacientes que cursen con brotes de la enfermedad en la cual, los cuadros pueden incluso producir paraplejías con vejiga e intestino neurogénico (por mielitis) o incluso verdaderas cuadriparesias severas con afectación a las actividades instrumentales de la vida diaria, para el manejo integral de la etapa aguda y de servicios externos, para el paciente ambulatorio con servicios de terapia física y ocupacional, de lenguaje, psicología y trabajo social.

\section{Consideraciones generales de rehabilitación actual de la esclerosis múltiple}

Tomando en cuenta que dentro de los padecimientos que afectan al sistema nervioso central la esclerosis múltiple, indica Neiger (2004), es la tercera causa de discapacidad en adultos jóvenes que se pueden ver afectados desde la segunda hasta la quinta década de la vida, y resaltando que generalmente afecta al individuo en la época de mayor productividad, es importante hacer un diagnóstico rehabilitatorio integral que involucre desde los aspectos clínicos particulares de cada caso, tipo de esclerosis y evolución, así como limitaciones y necesidades tanto físicas, psicológicas, sociales y familiares, para estar en condiciones de ofrecer el mejor tratamiento rehabilitatorio integral que proporcione terapia física, ocupacional, psicológica, social, recreativa y de integración con redes de apoyo familiar, considerando que es un padecimiento con expectativas de vida de mediano a largo plazo, para mejorar la propuesta de calidad de vida del paciente neurológico, de allí que un programa de rehabilitación que se inicia en las primeras fases de la enfermedad puede mejorar el estado general del paciente, tanto físico como emocional, y prevenir complicaciones, además de contribuir a la educación en salud de la persona afectada y sus familiares. La Esclerosis Múltiple es una enfermedad crónica que no tiene cura, con lo cual se 
recomienda que la persona que empieza a hacer rehabilitación lo haga de forma constante y disciplinada.

\section{Materiales y métodos}

La metodología fue de tipo observacional y descriptivo de campo. La aplicación del tratamiento rehabilitador se realizó en 30 pacientes con diagnóstico de Esclerosis Múltiple previamente confirmada por el neurólogo, atendidos en el Servicio de Medicina Física y Rehabilitación del Hospital de Especialidades Eugenio Espejo. Para su tratamiento se solicitó el consentimiento informado a los pacientes y familias que desearon participar en el estudio sobre la eficacia del tratamiento neuro - rehabilitador integral para mejorar la calidad de vida en pacientes con Esclerosis Múltiple. Los criterios de inclusión, eran pacientes con edades de adultez mayores a 20 años. Los datos se registraron en una lista de coteo, para finalmente aplicarle una estadística de tipo porcentual y de diferencias significativas entre los parámetros de la calidad de vida.

\section{Discusión y Resultados.}

Posterior al estudio de las características del sexo y edad, se pudo constatar que el $63 \%$ del total de los pacientes eran del sexo femenino, y el 85\% indicaron edades entre 28 y 52 años posteriores a su diagnóstico.

Se evidenció mayor cantidad de pacientes con formas clínicas progresivas, de los cuales el $64 \%$ eran trabajadores jubilados.

El 75,4 \% de los pacientes con EM presentaron la forma clínica remisión-recaída (EMRR), un 9 $\%$ primaria-progresiva (EMPP) y un 16,6\% secundaria-progresiva (EMSP).

En los pacientes tratados con diagnósticos de Esclerosis Múltiple recurrente-remitente (EMRR), el proceso de rehabilitación se realizaba por periodos determinados por el médico tratante, solo durante los periodos de recaídas, permitiéndoles mantener activos en sus trabajos.

Se evidencio mejorías en los pacientes con este padecimiento después de un programa de neurorehabilitación integral, independiente de la modalidad definidas de presentación para el inicio de sus terapias y la evolución de la EM, manifiestos en una mejora de su condición física y con factores relacionados a su calidad de vida. 
Cabe señalar, que se comprobó que la satisfacción sexual y el daño visual fueron los componentes de la calidad de vida que no presentaron diferencias significativas posteriores a las terapias de rehabilitación integral. Sin embargo, no así para la fatiga y el sueño que evidenciaron una correlación negativa estadísticamente significativa entre todos los componentes de la calidad de vida.

\section{Conclusiones.}

Se reveló predominio del sexo femenino entre los pacientes con EM investigados, y de las edades entre 28 y 52 años posteriores a su diagnóstico.

Se indicó mejoría para los componentes: fatiga y sueño, dentro de los evaluados en la calidad de vida posterior a las terapias de rehabilitación integral, fundamentalmente en una mejora de su condición física.

Recibieron un proceso de rehabilitación por periodos determinados por el médico tratante, y solo durante los periodos de recaídas en pacientes tratados con diagnósticos de EMRR.

\section{Referencias Bibliográficas}

Terré. Boliart, F. Orient y López. (2007). Tratamiento rehabilitador en la Esclerosis Múltiple. REV NEUROL.

Neiger H. (2004). Estiramientos Analíticos Manuales. Técnicas Pasivas. 1a. ed. Madrid: Editorial Médica Panamericana, SA.

Plaja J. Medicina de Rehabilitación Basada en la Evidencia. 2a. ed. Madrid: Interamericana de España; 2002.

Ruiz J, Martín MC y García R. (2006). Procedimientos y Técnicas de Enfermería. Madrid: Editorial ROL; 2006.

EME (2013). Esclerosis Múltiple España. Las claves de la rehabilitación en Esclerosis Múltiple • Recuperado en: https://www.esclerosismultiple.com/369/. 